\title{
Association between four SNPs on chromosome 9p21 and myocardial infarction is replicated in an Italian population
}

\author{
Gong-Qing Shen $\cdot$ Shaoqi Rao $\cdot$ Nicola Martinelli $\cdot$ Lin Li $\cdot$ Oliviero Olivieri · \\ Roberto Corrocher · Kalil G. Abdullah · Stanley L. Hazen · Jonathan Smith • \\ John Barnard · Edward F. Plow · Domenico Girelli · Qing K. Wang
}

Received: 18 October 2007 / Accepted: 14 November 2007/Published online: 8 December 2007

(C) The Japan Society of Human Genetics and Springer 2007

\begin{abstract}
Genome-wide single nucleotide polymorphism (SNP) association studies recently identified four SNPs (rs10757274, rs2383206, rs2383207, and rs10757278) on chromosome 9p21 that were associated with coronary artery disease (CAD) and myocardial infarction (MI) in Caucasian populations from northern Europe and North America. Our aim was to determine whether these SNPs were associated with MI in a southern Europe/Mediterranean population. We employed a case-control association design involving 416 MI patients and 308 non-MI controls from Italy. Significant allelic association was identified between all four
\end{abstract}

G.-Q. Shen, S. Rao, N. Martinelli, L. Li contributed equally to this work.

G.-Q. Shen · S. Rao - L. Li · E. F. Plow · Q. K. Wang $(\square)$

Department of Molecular Cardiology,

Center for Cardiovascular Genetics,

Department of Cardiovascular Medicine,

Cleveland Clinic, 9500 Euclid Ave.,

Cleveland, OH 44195, USA

e-mail: wangq2@ccf.org

N. Martinelli · O. Olivieri · R. Corrocher · D. Girelli $(\bowtie)$

Department of Clinical and Experimental Medicine,

University of Verona, 37134 Verona, Italy

e-mail: domenico.girelli@univr.it

K. G. Abdullah · S. L. Hazen · J. Smith · J. Barnard ·

E. F. Plow · Q. K. Wang

Cleveland Clinic Lerner College of Medicine,

Cleveland Clinic, Cleveland, $\mathrm{OH}$, USA

S. L. Hazen · J. Smith

Department of Cell Biology, Lerner Research Institute,

Cleveland Clinic, Cleveland, OH, USA

J. Barnard

Department of Quantitative Health Sciences,

Cleveland Clinic, Cleveland, OH, USA
SNPs and MI. The association remained significant after adjusting for covariates for MI $(P=0.007-0.029)$. One risk haplotype (GGGG; $P=0.028$ ) and one protective haplotype (AAAA; $P=0.047$ ) were identified. Genotypic association analysis demonstrated that the SNPs conferred susceptibility to MI most likely in a dominant model $(P=0.0007-0.013)$. When the case cohort was divided into a group of MI patients with a family history $(n=248)$ and one group without it $(n=168)$, the positive, significant association was identified only in the group with the family history. These results indicate that chromosome 9p21 confers risk for development of MI in an Italian population.

Keywords Coronary artery disease $\cdot$ Myocardial infarction - Single nucleotide polymorphisms (SNP) . Association study · Chromosome 9p21

\section{Introduction}

Genome-wide single nucleotide polymorphism (SNP) association studies are a recently developed strategy that has proved to be effective in identifying genetic factors for common, complex disease traits (Abecasis et al. 2007) such as diabetes (Saxena et al. 2007), obesity (Herbert et al. 2006), coronary artery disease (CAD), and myocardial infarction (MI) (Wellcome Trust Case Control Consortium 2007; Ozaki K et al. 2006). CAD, including its acute complication of MI, is the leading cause of mortality worldwide (Thom et al. 2006). Genetic factors have been defined as important risk contributors for the pathogenesis of CAD and MI (Wang 2005; Topol et al. 2006), but the responsible molecular genetic determinants remain largely unidentified.

Very recently, independent genome-wide SNP association studies identified four SNPs on chromosome 9p21 
associated with CAD and MI in several Caucasian cohorts, mainly from northern Europe and/or of northern European descent (McPherson et al. 2007; Helgadottir et al. 2007). McPherson et al. (2007) screened 100,000 SNPs throughout the genome and found two SNPs (rs10757274 and rs2383206) on chromosome 9p21 associated with CAD in a Canadian population and further replicated these findings in five other Caucasian cohorts from Denmark and the United States. Helgadottir et al. (2007) tested 305,953 SNPs for their association with MI and confirmed the association between MI and the same 9p21 region with two nearby SNPs (rs2383207 and rs10757278) using an Icelandic population, followed by replication of the findings in independent United States cohorts. The same genetic locus was identified by a genome-wide association study involving 1,926 cases and 3,000 controls from a British population by the Wellcome Trust Case Control Consortium (Wellcome Trust Case Control Consortium 2007) and replicated in a German population (Samani et al. 2007).

It is increasingly recognized that many independent replication studies are needed to unequivocally establish a valid genotype-phenotype association across diversities of human populations before the findings are extended to clinical settings and to the costly follow-up studies that are needed to identify causal genetic variants (Chanock et al. 2007). In particular, for a small- to medium-sized genotype-phenotype relationship with allelic risk odds ratios in the range of 1.25 , such as the association between the four SNPs on chromosome 9p21 and CAD/MI (Wellcome Trust Case Control Consortium 2007; Helgadottir et al. 2007), replications in as many populations as possible are essential for establishing the credibility of the locus-specific contribution. Therefore, we carried out a case-control association study in an Italian population. The goal of the study was to determine whether the chromosome 9p21 SNPs are associated with MI in the Italian population and to procure the wider genetic inferences of the locus effects across Caucasians of different geographic origins and life styles. Our study used a total of 416 well-defined, unrelated MI patients and 308 normal controls. All study subjects were Caucasians, although from a different geographic region (southern Europe/Mediterranean) compared with those in the studies previously reported. We found that chromosome 9p21 SNPs were also associated with MI in this southern European/Mediterranean population.

\section{Materials and methods}

Study subjects

For the case-control study, 416 unrelated MI adult patients and 308 normal controls were included from individuals participating in the Verona Heart Project. Of the 416 cases, 248 patients were individuals with a family history of MI and were selected for further study. The criteria for diagnostics and ascertainment have been described previously (Girelli et al. 1998; Girelli et al. 2000). In brief, the patients had angiographically proven CAD and the diagnosis of previous MI based on medical history, documentation of abnormal electrocardiograms, and enzymatic abnormalities. Controls had completely normal coronary arteries and were evaluated by coronary angiography for reasons other than CAD, mainly valvular heart disease. These controls were also required to have neither history nor clinical or instrumental evidence of atherosclerosis in vascular districts beyond the coronary bed. Both MI patients and controls came from the same geographical area, with a similar socioeconomic and ethnic background. At the time of blood sampling, a complete clinical history including cardiovascular risk factors such as smoking and hypertension was collected in all participating subjects. Hypertension was defined as blood pressure $\geq 140 /$ $90 \mathrm{mmHg}$ and/or the use of antihypertensive medication. The clinical characteristics of MI cases and normal controls are shown in Table 1.

As expected, clinical profiles for both groups were characteristic for subjects with the referred diagnostic groups, showing significant differences in several clinical or biochemical measures. This study was approved by the local institutional review boards on human subject research. Written informed consent was obtained from all participants. Whole blood was drawn from each participant, and

Table 1 Clinical characteristics of the Italian myocardial infarction patients and normal controls

\begin{tabular}{lllr}
\hline Characteristic & $\begin{array}{l}\text { Case } \\
(n=416)\end{array}$ & $\begin{array}{l}\text { Control } \\
(n=308)\end{array}$ & \multicolumn{1}{c}{$\begin{array}{l}P \\
\text { value }^{\mathrm{a}}\end{array}$} \\
\hline Gender (M/F) & $348 / 68$ & $214 / 94$ & $<0.001$ \\
Age (years) & $60.0 \pm 10.1$ & $58.1 \pm 12.6$ & 0.036 \\
Smoking (\%) & 71.1 & 45.1 & $<0.001$ \\
BMI $\left(\mathrm{kg} / \mathrm{m}^{2}\right)$ & $26.5 \pm 3.3$ & $25.3 \pm 3.4$ & $<0.001$ \\
Hypertension (\%) & 62.4 & 34.3 & $<0.001$ \\
Diabetes $(\%)$ & 23.4 & 12.6 & $<0.001$ \\
Total cholesterol (mmol/l) & $5.8 \pm 1.2$ & $5.5 \pm 1.1$ & $<0.001$ \\
LDL cholesterol (mmol/l) & $4.0 \pm 1.0$ & $3.6 \pm 0.9$ & $<0.001$ \\
Triglyceride (mmol/l) & $2.0 \pm 1.1$ & $1.5 \pm 0.7$ & $<0.001$ \\
\hline
\end{tabular}

$\overline{\text { Data are shown as mean } \pm \text { standard deviation, unless otherwise }}$ indicated

$B M I$ body mass index, $L D L$ low-density lipoprotein

a Continuous data were tested using two-tailed Student's $t$ test, and categorical data were tested using a chi-square test (with $d f=1$ ) for difference between case (patient) and control (normal) groups

b Age at onset for cases and age at examination for controls 
genomic DNA was isolated from the blood using standard protocols.

\section{Genotyping of SNPs}

The ABI PRISM 7900HT Sequence Detection System was used to perform SNP genotyping using the $5^{\prime}$ nuclease allelic discrimination assay (TaqMan Assay). The TaqMan Assay kit (Assays-on-Demand) was purchased from Applied Biosystems (Foster City, CA, USA). It included the forward target-specific polymerase chain reaction (PCR) primer, the reverse primer, and the TaqMan MGB probes labeled with two special dyes: FAM and VIC. Genotyping was performed in a 5 - $\mu$ l volume containing $2.5 \mu \mathrm{l}$ of TaqMan Universal PCR Master Mix, $0.125 \mu \mathrm{l}$ of $40 \times$ TaqMan MGB Assay Mix, and $25 \mathrm{ng}$ of genomic DNA. Automatic allele calling, with the default settings (the quality value of auto caller $\geq 95.0$ ), was carried out by ABI PRISM 7900HT data collection and analysis software version 2.1. The quality for SNP genotyping was assured by independently replicating the allelic calls of a number of randomly selected samples or by direct DNA sequence analysis of 24 samples.

\section{Statistical analysis}

A statistical power analysis was performed using a free program (Dupont and Plummer 1990) for power and sample size computations for case-control design, and both populations were determined able to provide statistical power. Allelic association of an SNP with a disease trait was assessed using the Pearson's $2 \times 2$ contingency table chi-square test implemented using SAS Ver 9.00 (SAS Institute Inc., Cary, NC, USA) or Haploview version 3.0 (http://www.broad.mit.edu/mpg/haploview/). Odds ratios and $95 \%$ confidence intervals (CI) were estimated using SAS Ver 9.00. Multivariate logistic regression analysis was performed using SAS Ver 9.00 to test relationships between SNPs and MI accounting to the covariates previously described [gender, age, smoking, body mass index (BMI), hypertension, diabetes, total cholesterol, low-density lipoprotein (LDL) cholesterol, and triglyceride level]. Due to lack of data on high-density lipoprotein (HDL) for a majority of patients and controls, HDL was omitted from multivariate logistic regression analysis. Empirical $P$ values were also calculated using 100,000 Monte Carlo simulations, by the CLUMP program (http://www.mds. qmw.ac.uk/statgen/dcurtis/software.html). Haplotypes were estimated using PHASE software (http://www.stat. washington.edu/stephens/software.html). Pairwise linkage disequilibrium (LD) between SNPs was measured as
$\mathrm{D}^{\prime}$ and $r^{2}$ using Haploview version 3.0 (http://www.broad. mit.edu/mpg/haploview/). Haplotype data were then subject to permutation analysis (with 100,000 Monte Carlo simulations) using Haploview software. Four SNPs were tested for Hardy-Weinberg equilibrium among all MI patients and normal controls using a chi-square test with one degree of freedom. For all analyses, point-wise statistical significance was taken at $P \leq 0.05$ and multipletests-corrected significance was determined using the conservative Bonferroni method (Bland and Altman 1995) and by considering the correlated structure between the studied SNPs.

\section{Results}

Power analysis indicates that the recruited Italian cohort is sufficient to replicate the previous findings

Prior to implementation of this replication study, we performed a statistical power analysis using the PS program (Dupont and Plummer 1990) to verify whether the recruited samples could provide adequate power in replicating the association between the modest-effect-size SNPs and MI, which was established by the previous studies (McPherson et al. 2007; Helgadottir et al. 2007; Wellcome Trust Case Control Consortium 2007; Samani et al. 2007). Under the population parameter settings of the effect size of odd ratios of 1.25 and the allelic frequency of 0.45 derived from the previous reports (McPherson et al. 2007; Helgadottir et al. 2007; Wellcome Trust Case Control Consortium 2007; Samani et al. 2007), our sample with 416 well-characterized MI cases and 308 normal controls with documented evidence of angiographically normal coronary arteries and lack of MI can provide statistical powers of $90 \%$ and $74 \%$ at the nominal type I error rate of 0.05 and 0.01 , respectively, indicating that our Italian sample was sufficient to replicate the previous results if the SNPs conferred the same risk as observed in the prior populations.

Allelic association analysis replicates the association between the four SNPs on 9p21 and MI

Prior to statistical analysis, we compared our genotyping data for control subjects with the data for European Caucasians estimated by the HapMap project. The allelic and genotypic distributions for the two cohorts were remarkably similar, indicating that our control subjects were representative Caucasians. All SNPs did not significantly $(P>0.1)$ deviate from the Hardy-Weinberg equilibrium (Table 2). 
Table 2 Allelic association of four single nucleotide polymorphisms (SNPs) with myocardial infarction cases and controls in the Italian population

\begin{tabular}{|c|c|c|c|c|c|c|c|c|}
\hline \multirow[t]{2}{*}{ SNPs } & \multirow[t]{2}{*}{ Allele } & \multicolumn{2}{|c|}{ Frequency } & \multirow[t]{2}{*}{ OR $(95 \%$ CI $)$} & \multicolumn{4}{|c|}{$P$ value } \\
\hline & & Control & Case & & $\mathrm{HW}^{\mathrm{a}}$ & Observed $^{\mathrm{b}}$ & Adjusted $^{c}$ & Empirical $^{\mathrm{d}}$ \\
\hline rs10757274 & G & 0.523 & 0.578 & $1.25(1.01-1.55)$ & 0.61 & 0.037 & 0.019 & 0.040 \\
\hline rs2383206 & G & 0.557 & 0.622 & $1.31(1.06-1.62)$ & 0.23 & 0.014 & 0.024 & 0.016 \\
\hline rs 2383207 & G & 0.571 & 0.627 & $1.26(1.02-1.56)$ & 0.23 & 0.033 & 0.029 & 0.038 \\
\hline rs10757278 & $\mathrm{G}$ & 0.503 & 0.565 & $1.28(1.04-1.58)$ & 0.16 & 0.022 & 0.007 & 0.024 \\
\hline
\end{tabular}

$O R$ odds ratio, $C I$ confidence interval

${ }^{\text {a }} \mathrm{HW}, P$ value for Hardy-Weinberg disequilibrium test

b Observed, nominal $P$ value

c Adjusted, $P$ value obtained after adjustment for gender, age, smoking, body mass index, hypertension, diabetes, total cholesterol, low-density lipoprotein cholesterol, and triglyceride level

${ }^{\text {d }}$ Empirical, permutation $P$ value calculated using 100,000 Monte Carlo simulations

Initially, we carried out a standard allelic association analysis for all four SNPs and found their significant association with the MI phenotype $[P$-obs (nominal $P$ value $)=0.014-0.037 ;$ odds ratio $(\mathrm{OR})=1.25-1.31]$ (Table 2). Then, to account for the interference of potential confounding factors with our assessment of the relations between the SNPs and MI, we performed a multivariate logistic regression analysis and verified that the significant association remained after adjusting for age, gender, smoking, BMI, hypertension, diabetes, total cholesterol, LDL cholesterol, and triglyceride level (the $P$ values after adjusting, $P$-adj $=0.007-0.029$, Table 2 ). Finally, we implemented a permutation test, a technique that is often used to determine the statistical significance of the experimental outcome by randomizing the data many times and is particularly useful when the asymptotic test deviates greatly from the true model. We used the CLUMP program to perform 100,000 Monte Carlo simulations to calculate the empirical $P$ value. Again, empirical $P$ values were significant $(P$-emp $=0.016-0.04$, Table 2$)$. In regards to multiple testing, we found these SNPs to be in high LD (Fig. 1), and given the degree of linkage, accepted nominal $P$ values in lieu of Bonferroni adjustment (Bland and Altman 1995; Storey and Tibshirani 2003). Considering all these results together, this study provided solid evidence that the SNPs on chromosome 9p21 are associated with MI and replicated the findings of previous studies in the Italian population.

Identification of two haplotypes associated with MI in the Italian cohort

An extended SNP haplotype analysis was conducted, which may provide some insights into the relationship between SNP patterns and MI that is beyond what singlepoint SNP analysis can reveal. Haplotypes were estimated
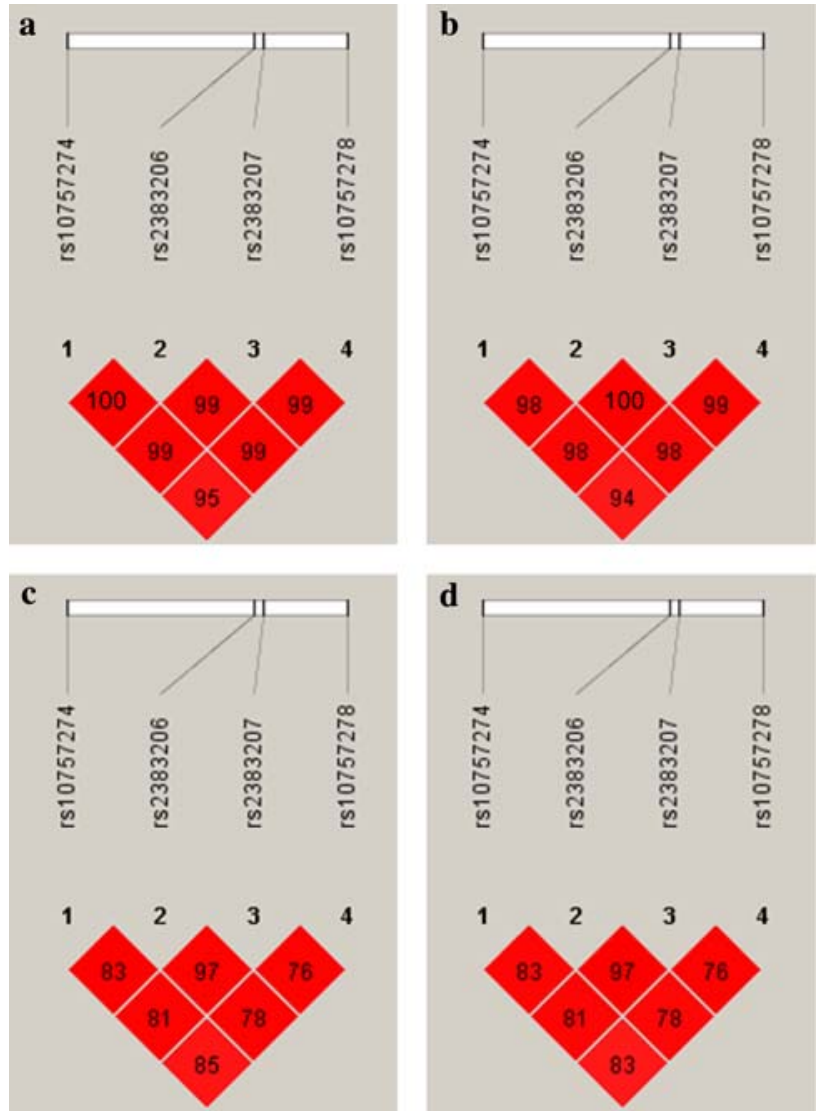

Fig. 1 Analysis of linkage disequilibrium (LD). LD patterns between four single nucleotide polymorphisms (SNPs), rs10757274, rs2383206, rs2383207 and rs10757278, were derived from the genotyping data from the Italian myocardial infarction (MI) (a, c) and control $(\mathbf{b}, \mathbf{d})$ populations, respectively. The pairwise correlation between SNPs was measured as $\mathrm{D}^{\prime}(\mathbf{a}, \mathbf{b})$ or $r^{2}(\mathbf{c}, \mathbf{d})$ and are shown $(\times 100)$ in each diamond

using an accelerated expectation-maximization algorithm, which gives highly accurate population frequency estimates of the phased haplotypes based on the maximum 
likelihood as determined from the unphased input (Barrett et al. 2005). Five haplotypes were identified in both cases and controls (Table 3). As the four SNPs on chromosome 9 p21 were highly correlated as revealed in Fig. 1, each haplotype was thus treated as a single variant (all the remaining haplotypes were collapsed into the alternative allele) to test its association with the disease trait. As shown in Table 3, the two most common haplotypes, GGGG and AAAA, showed strong associations in the

Table 3 Association analysis of haplotypes of four single nucleotide polymorphisms in the Italian population

\begin{tabular}{lrrlll}
\hline Haplotype & $\begin{array}{l}\text { Control } \\
(\%)\end{array}$ & $\begin{array}{l}\text { Case } \\
(\%)\end{array}$ & OR $(95 \%$ CI $)$ & \multicolumn{2}{l}{$P$ value } \\
\cline { 5 - 6 } & & & & Observed $^{\mathrm{a}}$ & Empirical $^{\mathrm{b}}$ \\
\hline GGGG & 49.6 & 55.3 & $1.27(1.03-1.56)$ & 0.028 & 0.028 \\
AAAA & 42.4 & 37.0 & $0.80(0.65-1.00)$ & 0.047 & 0.049 \\
AGGA & 3.1 & 3.5 & $1.14(0.63-2.04)$ & 0.678 & 0.765 \\
GGGA & 2.7 & 2.3 & $0.83(0.42-1.64)$ & 0.566 & 0.603 \\
AGGG & 1.0 & 1.1 & $1.11(0.39-3.13)$ & 0.855 & 1.000 \\
\hline
\end{tabular}

$O R$ odds ratio, $C I$ confidence interval

a Observed, nominal $P$ value

b Empirical, permutation $P$ value calculated using 100,000 Monte Carlo simulations opposite directions with MI in the population. Haplotype GGGG conferred a significant risk effect $(P$-obs $=0.028$; $P$-emp $=0.028 ; \quad$ OR $=1.27 ; \quad 95 \% \quad$ CI $=1.03-1.56)$, whereas the AAAA haplotype conferred protection of MI $(P$-obs $=0.047 ; \quad P$-emp $=0.049 ; \quad$ OR $=0.80 ; 95 \% \quad \mathrm{CI}=$ $0.65-1.00)$.

Genotypic association analysis suggests that the putative genetic model involved in development of $\mathrm{MI}$ is dominant

To further investigate how SNP alleles (or haplotypes) interact in conferring genetic risk to $\mathrm{MI}$, we further conducted genotypic association analysis assuming three common genetic models (dominant, recessive, and additive inheritance). The results for the four SNPs and their haplotypes (considering two most common ones as alternative alleles) consistently supported that either SNPs directly exert an effect or the linked functional gene impacts the disease trait in a dominant model (without adjustment for covariates, $P=0.004-0.034$; after adjustment for covariates, $P=0.0007-0.013$ ) or less likely the additive model (without adjustment for covariates, $P=0.016-0.063$; after adjustment for covariates, $P=0.007-0.025$ ) (Table 4 ).

Table 4 Genetic models of the four single nucleotide polymorphisms (SNPs) in the Italian population

\begin{tabular}{|c|c|c|c|c|c|}
\hline \multirow[t]{2}{*}{ Variant } & \multirow[t]{2}{*}{ Model } & \multicolumn{2}{|c|}{ Without adjustment $^{\mathrm{a}}$} & \multicolumn{2}{|c|}{ With adjustment $^{\mathrm{b}}$} \\
\hline & & $P$ & OR $(95 \% \mathrm{CI})^{\mathrm{c}}$ & $P$ & OR $(95 \% \mathrm{CI})$ \\
\hline \multicolumn{6}{|l|}{ Single SNP } \\
\hline \multirow[t]{3}{*}{ rs 10757274} & Dominance & 0.032 & $1.50(1.0-2.2)$ & 0.007 & $1.79(1.2-2.7)$ \\
\hline & Recessive & 0.194 & $1.24(0.9-1.7)$ & 0.082 & $1.38(1.0-2.0)$ \\
\hline & Additive & 0.038 & $1.25(1.0-1.5)$ & 0.007 & $1.39(1.1-1.8)$ \\
\hline \multirow[t]{3}{*}{ rs 2383206} & Dominance & 0.018 & $1.61(1.1-2.4)$ & 0.013 & $1.75(1.1-2.8)$ \\
\hline & Recessive & 0.096 & $1.31(0.9-1.8)$ & 0.098 & $1.35(1.0-1.9)$ \\
\hline & Additive & 0.016 & $1.30(1.0-1.6)$ & 0.014 & $1.35(1.1-1.7)$ \\
\hline \multirow[t]{3}{*}{ rs2383207 } & Dominance & 0.034 & $1.53(1.0-2.3)$ & 0.013 & $1.79(1.1-2.8)$ \\
\hline & Recessive & 0.159 & $1.25(0.9-1.7)$ & 0.106 & $1.33(0.9-1.9)$ \\
\hline & Additive & 0.037 & $1.25(1.0-1.5)$ & 0.015 & $1.35(1.1-1.7)$ \\
\hline \multirow[t]{3}{*}{ rs 10757278} & Dominance & 0.004 & $1.70(1.2-2.4)$ & 0.0007 & $2.04(1.4-3.0)$ \\
\hline & Recessive & 0.361 & $1.17(0.8-1.6)$ & 0.305 & $1.22(0.8-1.8)$ \\
\hline & Additive & 0.023 & $1.28(1.0-1.6)$ & 0.009 & $1.38(1.1-1.8)$ \\
\hline \multicolumn{6}{|l|}{ Haplotype } \\
\hline \multirow[t]{3}{*}{ GGGG versus AAAA } & Dominance & 0.022 & $1.59(1.1-2.4)$ & 0.006 & $1.90(1.2-3.0)$ \\
\hline & Recessive & 0.382 & $1.16(0.8-1.6)$ & 0.280 & $1.24(0.8-1.8)$ \\
\hline & Additive & 0.063 & $1.23(1.0-1.6)$ & 0.025 & $1.34(1.0-1.7)$ \\
\hline
\end{tabular}

$O R$ odds ratio, $C I$ confidence interval

${ }^{\text {a }} P$ values were obtained from logistic regression modeling where the predictor is a single SNP

${ }^{\mathrm{b}} P$ values were obtained from logistic regression modeling after adjustment for gender, age, smoking, body mass index, hypertension, diabetes, total cholesterol, low-density lipoprotein cholesterol, and triglyceride level 
SNPs on chromosome 9p21 confer risk to MI with a family history

To determine whether the four SNPs in this study were associated with MI patients with a family history, we analyzed a subset of the cohort $(n=248)$ with a positive family history of MI. Results showed that the association of the four SNPs with MI with a positive family history was significant for association with MI $(P=0.005-0.028)$ (Table 5). The association with MI was not significant if only 168 MI patents without a family history were analyzed as the case cohort $(P=0.148-0.204)$. These results suggest that a stronger association for the four 9p21 SNPs exists for MI patients with a family history than for those without it.

\section{Discussion}

Given the fast pace of genomic research, the ability to properly analyze reported associations between SNPs and their associated phenotype has become a necessity (Chanock et al. 2007). As such, we first and foremost took significant steps to assure that our study was conformant to the standards outlined by the National Cancer Institute (NCI) and the National Human Genome Research Institute (NCI-NHGRI) Working Group on Replication in Association Studies for establishing positive replication (Chanock et al. 2007).

The recruited Italian MI and control samples, originally used in several studies of candidate genes (Girelli et al. 1998; Girelli et al. 2000), were carefully designed. In addition to the strict criteria used to define the MI and the normal physiological phenotype (for details, see Girelli et al. 2000), an intention for matching cases and controls

Table 5 Association analysis of the four 9p21 single nucleotide polymorphisms (SNPs) with myocardial infarction with and without family history

\begin{tabular}{|c|c|c|c|c|}
\hline \multirow[t]{2}{*}{ SNPs } & \multicolumn{2}{|c|}{$\begin{array}{l}\text { Without family } \\
\text { history }(n=168)\end{array}$} & \multicolumn{2}{|c|}{$\begin{array}{l}\text { With family } \\
\text { history }(n=248)\end{array}$} \\
\hline & $P$ & OR $(95 \% \mathrm{CI})$ & $P^{\mathrm{a}}$ & OR $(95 \% \mathrm{CI})$ \\
\hline rs10757274 & 0.204 & $1.19(0.9-1.6)$ & 0.015 & $1.41(1.1-1.9)$ \\
\hline rs2383206 & 0.149 & $1.22(0.9-1.6)$ & 0.018 & $1.40(1.1-1.8)$ \\
\hline rs 2383207 & 0.148 & $1.22(0.9-1.6)$ & 0.028 & $1.36(1.0-1.8)$ \\
\hline rs10757278 & 0.193 & $1.19(0.9-1.6)$ & 0.005 & $1.47(1.1-1.9)$ \\
\hline
\end{tabular}

$P$ value all compared with 308 normal controls

$O R$ odds ratio, $C I$ confidence interval

${ }^{a} P$ values were obtained from logistic regression modeling after adjustment for gender, age, smoking, body mass index, hypertension, diabetes, total cholesterol, low-density lipoprotein cholesterol, and triglyceride level was applied to reduce potential confounding due to marked differences between the two designed groups. Although statistically significant differences for most of MI risk factors were observed, discrepancies between means and variances of case and control groups were relatively small after matching, which may largely reflect the inherent characteristics for the two referred populations.

Our study analyzed well-characterized Italian MI patients and controls and not only replicated the findings of the four SNPs on chromosome 9p21 that were associated with CAD and MI established previously by genomewide association analysis, but also provided several novel findings that are relevant to this locus. The major findings include: (1) four SNPs in one strong LD block showed significant allelic or genotypic associations with $\mathrm{MI}$ in an Italian population; (2) positive associations remained valid after adjustment for other risk factors for MI (age, gender, smoking, BMI, hypertension, diabetes, total cholesterol, LDL cholesterol, and triglyceride level); (3) both risk and protective haplotypes conferred susceptibility to MI; (4) dominant inheritance was the most likely allelic interaction model by comparing different genetic models, indicating that SNPs may be linked to a disease-susceptibility gene whose one copy is sufficient to increase the risk of MI; (5) when the case cohort was divided into one group of MI patients with a family history $(n=248)$ and one without $(n=168)$, the association was identified only in the group with the family history; (6) this study is the first replication of associations of the four SNPs on 9p21 with MI in a southern European/Mediterranean (Italian) population.

We should recognize the limitations of this study or any single human genetic study because of the sample size, which is often fixed, and the genetic complexities of the studied disease (e.g., involvement of multiple small- to modest-sized effects of genes and polygenic and environmental backgrounds). As such, the genetic parameter estimates (ORs, risk, and allelic or genotypic frequencies) for the Italian population may be biased and not reach the adequate precision for population genetic studies or may not be used in the clinic. However, based on the consistent risk estimates of SNPs from previous studies and the one reported here, in addition to stringent analysis that includes haplotypic and model-based significance, we are confident that the risk estimation we report is valid.

In conclusion, we found associations between four SNPs on chromosome 9p21 and MI in southern European/Mediterranean populations, identifying both risk and protective haplotypes and a model of genetic inheritance.

Acknowledgments This work was supported by an NIH grant P50 HL77107 (EFP, QKW, JS, SH, JB), by grants from the Italian Ministry of University and Research (grant no. 2005/065152), and the 
Cariverona Foundation, Verona, Italy (DG), the Cleveland Clinic Lerner College of Medicine (KGA), China National 863 Scientific Program (2006AA02Z476) (QKW), and an American Heart Association Established Investigator award (QKW).

\section{References}

Abecasis G, Tam PK, Bustamante CD, Ostrander EA, Scherer SW, Chanock SJ, Kwok PY, Brookes AJ (2007) Human Genome Variation 2006: emerging views on structural variation and large-scale SNP analysis. Nat Genet 39:153-155

Barrett JC, Fry B, Maller J, Daly MJ (2005) Haploview: analysis and visualization of LD and haplotype maps. Bioinformatics 21:263265

Bland JM, Altman DG (1995) Multiple significance tests: the Bonferroni method. BMJ 310:170

Chanock SJ, Manolio T, Boehnke M, Boerwinkle E, Hunter DJ, Thomas G, Hirschhorn JN, Abecasis G, Altshuler D, BaileyWilson JE, Brooks LD, Cardon LR, Daly M, Donnelly P, Fraumeni JF Jr, Freimer NB, Gerhard DS, Gunter C, Guttmacher AE, Guyer MS, Harris EL, Hoh J, Hoover R, Kong CA, Merikangas KR, Morton CC, Palmer LJ, Phimister EG, Rice JP, Roberts J, Rotimi C, Tucker MA, Vogan KJ, Wacholder S, Wijsman EM, Winn DM, Collins FS (2007) Replicating genotype-phenotype associations. Nature 447:655-660

Dupont WD, Plummer WD Jr (1990) Power and sample size calculations. A review and computer program. Control Clin Trials 11:116-128

Girelli D, Friso S, Trabetti E, Olivieri O, Russo C, Pessotto R, Faccini G, Pignatti PF, Mazzucco A, Corrocher R (1998) Methylenetetrahydrofolate reductase C677T mutation, plasma homocysteine, and folate in subjects from northern Italy with or without angiographically documented severe coronary atherosclerotic disease: evidence for an important genetic-environmental interaction. Blood 91:4158-4163

Girelli D, Russo C, Ferraresi P, Olivieri O, Pinotti M, Friso S, Manzato F, Mazzucco A, Bernardi F, Corrocher R (2000) Polymorphisms in the factor VII gene and the risk of myocardial infarction in patients with coronary artery disease. N Engl J Med 343:774-780

Helgadottir A, Thorleifsson G, Manolescu A, Gretarsdottir S, Blondal T, Jonasdottir A, Jonasdottir A, Sigurdsson A, Baker A, Palsson A, Masson G, Gudbjartsson DF, Magnusson KP, Andersen K, Levey AI, Backman VM, Matthiasdottir S, Jonsdottir T, Palsson S, Einarsdottir H, Gunnarsdottir S, Gylfason A, Vaccarino V, Hooper WC, Reilly MP, Granger CB, Austin H, Rader DJ, Shah SH, Quyyumi AA, Gulcher JR, Thorgeirsson G, Thorsteinsdottir U, Kong A, Stefansson K (2007) A common variant on chromosome 9p21 affects the risk of myocardial infarction. Science 316:1491-1493

Herbert A, Gerry NP, McQueen MB, Heid IM, Pfeufer A, Illig T, Wichmann HE, Meitinger T, Hunter D, Hu FB, Colditz G, Hinney A, Hebebrand J, Koberwitz K, Zhu X, Cooper R, Ardlie K, Lyon H, Hirschhorn JN, Laird NM, Lenburg ME, Lange C,
Christman MF (2006) A common genetic variant is associated with adult and childhood obesity. Science 312:279-283

McPherson R, Pertsemlidis A, Kavaslar N, Stewart A, Roberts R, Cox DR, Hinds DA, Pennacchio LA, Tybjaerg-Hansen A, Folsom AR, Boerwinkle E, Hobbs HH, Cohen JC (2007) A common allele on chromosome 9 associated with coronary heart disease. Science 316:1488-1491

Ozaki K, Sato H, Iida A, Mizuno H, Nakamura T, Miyamoto Y, Takahashi A, Tsunoda T, Ikegawa S, Kamatani N, Hori M, Nakamura Y, Tanaka T (2006) A functional SNP in PSMA6 confers risk of myocardial infarction in the Japanese population. Nat Genet 38:921-925

Samani NJ, Erdmann J, Hall AS, Hengstenberg C, Mangino M, Mayer B, Dixon RJ, Meitinger T, Braund P, Wichmann HE, Barrett JH, König IR, Stevens SE, Szymczak S, Tregouet DA, Iles MM, Pahlke F, Pollard H, Lieb W, Cambien F, Fischer M, Ouwehand W, Blankenberg S, Balmforth AJ, Baessler A, Ball SG, Strom TM, Braenne I, Gieger C, Deloukas P, Tobin MD, Ziegler A, Thompson JR, Schunkert H, WTCCC and the Cardiogenics Consortium (2007) Genomewide association analysis of coronary artery disease. N Engl J Med 357:443-453

Saxena R, Voight BF, Lyssenko V, Burtt NP, de Bakker PI, Chen H, Roix JJ, Kathiresan S, Hirschhorn JN, Daly MJ, Hughes TE, Groop L, Altshuler D, Almgren P, Florez JC, Meyer J, Ardlie K, Bengtsson Boström K, Isomaa B, Lettre G, Lindblad U, Lyon HN, Melander O, Newton-Cheh C, Nilsson P, Orho-Melander M, Råstam L, Speliotes EK, Taskinen MR, Tuomi T, Guiducci C, Berglund A, Carlson J, Gianniny L, Hackett R, Hall L, Holmkvist J, Laurila E, Sjögren M, Sterner M, Surti A, Svensson M, Svensson M, Tewhey R, Blumenstiel B, Parkin M, Defelice M, Barry R, Brodeur W, Camarata J, Chia N, Fava M, Gibbons J, Handsaker B, Healy C, Nguyen K, Gates C, Sougnez C, Gage D, Nizzari M, Gabriel SB, Chirn GW, Ma Q, Parikh H, Richardson D, Ricke D, Purcell S (2007) Genome-wide association analysis identifies loci for type 2 diabetes and triglyceride levels. Science 316:1331-1336

Storey JD, Tibshirani R (2003) Statistical significance for genomewide studies. Proc Natl Acad Sci USA 100:9440-9445

Thom T, Haase N, Rosamond W, Howard VJ, Rumsfeld J, Manolio T, Zheng ZJ, Flegal K, O’Donnell C, Kittner S, Lloyd-Jones D, Goff DC Jr, Hong Y, Adams R, Friday G, Furie K, Gorelick P, Kissela B, Marler J, Meigs J, Roger V, Sidney S, Sorlie P, Steinberger J, Wasserthiel-Smoller S, Wilson M, Wolf $\mathrm{P}$, American Heart Association Statistics Committee, Stroke Statistics Subcommittee (2006) Heart disease and stroke statistics2006 update: a report from the American Heart Association Statistics Committee and Stroke Statistics Subcommittee. Circulation 113:e85-e151

Topol EJ, Smith J, Plow EF, Wang QK (2006) Genetic susceptibility to myocardial infarction and coronary artery disease. Hum Mol Genet 15:R117-R123

Wang Q (2005) Advances in the genetic basis of coronary artery disease. Curr Atheroscler Rep 7:235-241

Wellcome Trust Case Control Consortium (2007) Genome-wide association study of 14,000 cases of seven common diseases and 3,000 shared controls. Nature 447:661-678 